\title{
CONCENTRACIONES MÁXIMAS DE LACTATO SANGUÍNEO PRODUCIDOS EN COMBATES DE ESGRIMA DEPENDIENDO DEL TIPO DE ARMA
}

\section{MAXIMAL BLOOD LACTATE CONCENTRATIONS PRODUCED IN FENCING MATCHES GIVEN BY THE TYPE OF WEAPON}

\section{Bahamondes-Avila, Carlos ${ }^{1}$; Cárcamo-Oyarzún, Jaime ${ }^{2} \&$ Hernández-Mosqueira, Claudio $^{3}$}

\author{
${ }^{1}$ Escuela de Kinesiología, Laboratorio de Fisiología y Movimiento Humano, Universidad Mayor Sede Temuco. \\ ${ }^{2}$ Centro Trapananda, Universidad Austral de Chile, Campus Patagonia, Coyhaique, Chile. \\ ${ }^{3}$ Grupo de investigación en Ciencias de la Actividad Física, Salud y Deportes. Carrera Pedagogía en Educación Física. Universidad \\ Pedro de Valdivia, Campus Chillán, Chile.
}

BAHAMONDES-AVILA, C.; CÁRCAMO-OYARZÚN, J. \& HERNÁNDEZ-MOSQUEIRA, C. (2014).Concentraciones máximas de lactato sanguíneo producidos en combates de esgrima dependiendo del tipo de arma. Mot. Hum. 15(2): 116-123.

\section{RESUMEN}

Debido a la predominancia de movimientos breves y explosivos, la larga duración de los torneos oficiales, y las diferencias reglamentarias, técnicas y tácticas de cada una de las armas, no existe una homogeneidad de criterios en referencia a la contribución de los sistemas energéticos participantes en la esgrima. Determinar los niveles máximos de lactato sanguíneo que producen esgrimistas durante una competencia oficial, y determinar si existen diferencias según cada arma. Se realizó la medición de lactato a 24 esgrimistas adultos (ocho por cada una de las tres armas) luego de finalizado los combates de la fase de octavos de final de una competencia oficial de carácter Nacional. Los valores promedios obtenidos para las tres armas fueron de 2,62 mmol/lt. (espada: 2,46 mmol/lt; sable: 2,67 mmol/lt y florete: 2,73 mmol/lt.), no existiendo diferencias estadísticamente significativas entre las tres armas. Las concentraciones máximas de lactato en la fase de octavos de final de una competencia oficial indican una escasa participación del sistema anaeróbico láctico; además, el tipo de arma no influiría en las concentraciones de ácido láctico producidas en combate. Los resultados de este estudio sirven de herramienta para mejorar la aplicación de las cargas físicas y selección de métodos de entrenamiento, los que deben considerar la predominancia de los sistemas energéticos anaeróbico aláctico y aeróbico.

Palabras Claves: Lactato, Esgrima, Metabolismo energético

\section{ABSTRACT}

Due to the predominance of brief and explosive movements, the length of the official tournaments and the differences in regulations, techniques and tactics of each of the weapons, there's a lack of criteria homogeneity in reference to the contribution of the energy systems involved in fencing. The objective of this study is to determine the maximal levels of blood lactate produced by fencers during an official tournament, and determine if differences exist between each weapon. A lactate measurement was performed on 24 adult fencers (eight for each of the three weapons) after the end of their respective matches in the combats of the eighth finals in a official competition. The values from the averages obtained for the three weapons were that of 2,62 mmol/lt (sword: 2,46 mmol/lt; sable 2,67 mmol/lt and foil: 2,73 mmol/lt), without any statistical significant differences found between the three weapons. The maximal concentrations of lactate in the combats of the eighth finals in a official competition indicate a low participation of the anaerobic lactate system; furthermore, the type of weapon would not influence the maximum concentrations of lactic acid produced in combat. The results of this study can be used as a tool to improve on the application of physical burdens and selection of training methods, in particular, those which must take into account the anaerobic lactic and aerobic systems.

Key Words: Lactate, Fencing, Energetic Metabolism 
BAHAMONDES-AVILA, C.; CÁRCAMO-OYARZÚN, J. \& HERNÁNDEZ-MOSQUEIRA, C. (2014). Concentraciones máximas de lactato sanguíneo producidos en combates de esgrima dependiendo del tipo de arma. Mot. Hum. 15(2): 116-123.

\section{INTRODUCCION}

La esgrima es una de las pocas disciplinas deportivas que siempre se han disputado en la historia de los Juegos Olímpicos, estando presente desde los I Juegos Olímpicos en Atenas 1896 con las armas de florete y sable, y desde los Juegos Olímpicos de 1900 en Paris con la espada. Calificada como un deporte de combate entre dos tiradores, esta disciplina se basa en el principio de tocar al oponente sin ser tocado, compitiéndose en 3 tipos de armas diferentes: la espada, el sable y el florete, las cuales difieren reglamentariamente. En la espada está permitido tocar con la punta del arma en cualquier parte del cuerpo del adversario; en el sable se puede tocar con cualquier parte de la hoja (con la punta o con el filo), por arriba de la cintura del adversario; y en el florete, solo se permite tocar con la punta del arma en el área del tronco del adversario.

Otra característica importante de este deporte es la duración de los combates, la cual depende de la fase de la competencia. En la primera fase, que se realiza en grupos o "poules", los combates tienen una duración máxima de 3 minutos efectivos (cronometrados, es decir, cada vez que se detiene el combate, también se detiene el tiempo), terminándose antes si un tirador es tocado 5 veces. En las fases siguientes se utiliza el sistema de eliminación directa hasta llegar a la final, en donde se disputan combates hasta los 15 puntos, con una duración máxima de 9 minutos efectivos, divididos en 3 periodos de 3 minutos, con un minuto de pausa entre cada periodo. Además de estas pausas reglamentarias, dentro de estos períodos se producen pequeñas pausas luego de finalizada una acción. La duración de cada acción puede ser muy corta e intensa (menos de 1 segundo), o puede durar más de 60 segundos (a rendimiento submáximo), existiendo evidencias de que en promedio una acción duraría 5 segundos en florete y 15 segundos en espada, con una razón de trabajo:pausa de 1:1 en espada masculina, 1:3 en florete masculino y $2: 1$ en espada de femenina (Roi \& Bianchedi, 2008). En relación a la duración total de un torneo, conformado por varios combates durante las diversas fases, se puede llegar a competir hasta 11 horas (Roi \& Bianchedi, 2008)
La esgrima es un deporte en que predominan los movimientos breves y explosivos, por lo que las cualidades físicas más recurrentes son la fuerza explosiva y la velocidad de movimientos, sin embargo, por las características de la larga duración de los torneos y las fases intermitentes de recuperación (Bottoms, 2011; Roi \& Bianchedi, 2008), la resistencia aeróbica también juega un rol considerable dentro de las cualidades físicas de los esgrimistas (Iglesias \& Rodríguez, 2000; Wylde, Tan, \& O'Donoghue, 2013) ya que esta cualidad tendría directa relación con la capacidad de recuperación del sistema fosfágeno (Ryan, Southern, Reynolds, \& McCully, 2013; Tomlin \& Wenger, 2001). Por otro lado, el rendimiento deportivo en esgrima también tiene una alta dependencia de factores técnicos coordinativos, así como de procesos cognitivos relacionados con estrategias tácticas durante la competición (Iglesias, Irurtia, Marina, \& Carrasco); los que pueden verse afectados por la edad, sexo, características antropométricas, nivel de formación y técnica y sistemas tácticos utilizados en relación al adversario (Ghloum \& Hajji, 2011; Marginet et al., 2008; Roi \& Bianchedi, 2008)

Teniendo en cuenta estas características y considerando la intermitencia de acciones cortas pero muy intensas con una alta demanda del sistema fosfágeno, la fuente de suministro energético que predomina en la esgrima es la de tipo anaeróbico aláctico (Bottoms, 2011; Iglesias \& Rodríguez, 1995), sin embargo no existe la misma homogeneidad de criterios en referencia a la contribución de los sistemas anaeróbico láctico (Bottoms, 2011; Iglesias \& Rodríguez, 1995; Roi \& Bianchedi, 2008) y aeróbico (Ghloum \& Hajji, 2011; Weichenberger, Liu, \& Steinacker, 2012). En este contexto, conocer el comportamiento del lactato durante el ejercicio tiene importantes aplicaciones, tales como la valoración de la capacidad de resistencia, la evaluación de los efectos del entrenamiento y la prescripción de la intensidad del ejercicio (Faude, Kindermann, \& Meyer, 2009; Souza et al., 2012). En este sentido, para deportes que poseen características de intermitencia, se ha hecho muy común la medición del lactato sanguíneo como indicador de la necesidad metabólica; determinando intensidades a través de pruebas incrementales realizadas en treadmill o cicloergómetro. 
BAHAMONDES-AVILA, C.; CÁRCAMO-OYARZÚN, J. \& HERNÁNDEZ-MOSQUEIRA, C. (2014). Concentraciones máximas de lactato sanguíneo producidos en combates de esgrima dependiendo del tipo de arma. Mot. Hum. 15(2): 116-123.

Sin embargo, dada la naturaleza intermitente de los combates de esgrima, estos test incrementales no permiten obtener datos concretos de la demanda anaeróbica y aeróbica de esta disciplina (Weichenberger et al., 2012); por lo que valorar el comportamiento del lactato en pruebas de laboratorio entregaría solo una información parcial. Considerando que la exigencia de la esgrima es multifactorial, tanto por ser un deporte de oposición como por la naturaleza de cada una de las armas, para conocer los procesos fisiológicos asociados al metabolismo del lactato es necesario incluir variables que se producen solo en condiciones de competencia, incluyendo no tan solo las exigencias físicas propias de un combate, sino que también las condicionantes psicológicas y cognitivas, así como las características técnicas y tácticas específicas de cada arma (Weichenberger et al., 2012). Por ese motivo, los objetivos de este estudio son, por un lado, determinar los niveles máximos de lactato sanguíneo que producen los esgrimistas varones de la Federación Chilena de Esgrima durante una competencia oficial de carácter Nacional; y por otro, comparar esos valores entre cada una de las armas y así definir si las exigencias fisiológicas metabólicas difieren entre ellas.

\section{MATERIAL Y METODOS}

La muestra correspondió a 24 esgrimistas de la Categoría Adultos Varones (8 en el arma de espada, 8 en florete y 8 en sable), que participaron en el campeonato oficial válido por el ranking nacional, organizado por la Federación Chilena de Esgrima. La muestra fue constituida por los ganadores de los combates correspondientes a los octavos de final, es decir, los tiradores que clasificaron a cuartos de final. Preliminarmente, y teniendo en cuenta los principios éticos de investigación humana de la declaración de Helsinki, se solicitó a cada deportista la participación voluntaria, firmando para ello un consentimiento informado y contando además con la autorización de su cuerpo técnico y la de la comisión técnica de la Federación Chilena de Esgrima.

Previo a la toma de muestras a los ganadores de los octavos de final, se realizó un estudio piloto (datos de los autores no publicados) durante la fase de grupos (poules), para analizar el comportamiento de la cinética del lactato durante el combate, se realizó un protocolo siguiendo las recomendaciones planteadas por diversos autores (Gharbi et al., 2008; Menzies et al., 2010; Moxnes \& Sandbakk, 2012); esto es, evaluación previo al combate; inmediatamente al término del combate; transcurridos los minutos uno; tres y cinco de finalizado el combate, observándose que los valores máximos se encontraban un minuto después de terminado el combate; por ello, las muestras de esta investigación se tomaron transcurrido este período de tiempo.

Para la medición del lactato sanguíneo se utilizó un minifotómetro marca Dr Lange LP20 (Hach Lange, Düsseldorf, Alemania), que posee rangos de medición de 0 a $30 \mathrm{mmol} / \mathrm{lt}$, y reactivos Dr. Lange LKM 140 para lactato. Se siguieron las recomendaciones de diversos autores (Dassonville et al., 1998; Santhiago, Silva, Guglielmo, \& Higino, 2008), con una lanceta desechable y capilares con heparina se colectaron 10ul de sangre desde el lóbulo de la oreja, luego se depositó la muestra en los tubos reactivos para lactato y se introdujo en la celda fotosensible del minifotómetro. En todos los casos se respetó el proceso de limpieza y asepsia, utilizando técnicas de enfermería habituales.

Para el análisis estadístico de los datos se utilizó el programa IBM SPSS Statistics 19.0. Para determinar la normalidad de los datos se aplicó el test de Shapiro-Wilk. En el análisis estadístico descriptivo se presenta un perfil del conjunto de datos a través de los valores medios, desviación estándar y valores extremos (mínimo y máximo). Para el análisis estadístico inferencial se realizó un análisis de variancia con el método ANOVA para determinar si existen diferencias significativas entre las tres armas (espada, florete y sable), asumiéndose como significativas con un nivel $\alpha$ de $p \leq 0,05$. 
BAHAMONDES-AVILA, C.; CÁRCAMO-OYARZÚN, J. \& HERNÁNDEZ-MOSQUEIRA, C. (2014). Concentraciones máximas de lactato sanguíneo producidos en combates de esgrima dependiendo del tipo de arma. Mot. Hum. 15(2): 116-123.

\section{RESULTADOS}

En la Figura 1 se presentan los valores medios de la concentración de lactato sanguíneo obtenido en las tres armas, los que oscilan entre 2,46 y $2,73 \mathrm{mmol} / \mathrm{lt}$.

Figura 1: Media de la Concentración de Lactato producidas en combates de esgrima, según arma.

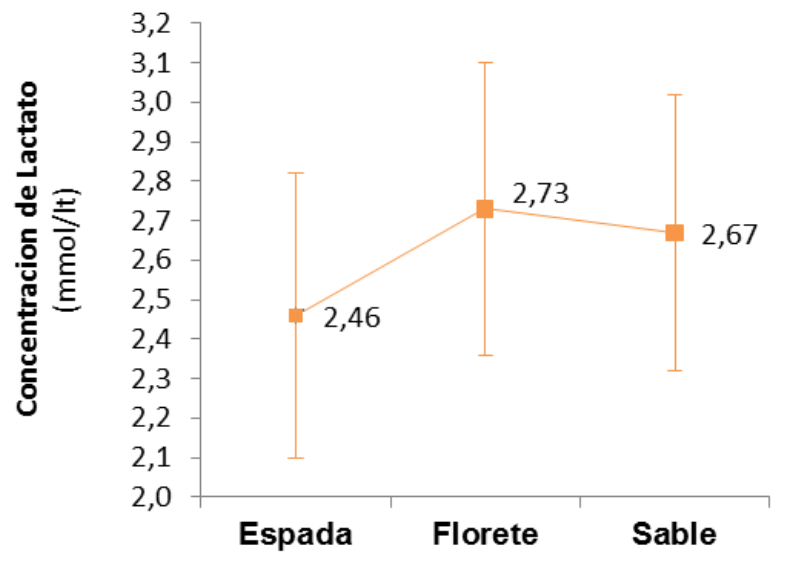

En la Tabla I se presentan la media, desviación estándar y valores máximos y mínimos de la concentración de lactato sanguíneo de cada una de las armas (espada, florete y sable), así como la media de todas las armas. Como se puede observar, la media de la concentración de lactato sanguíneo que considera a todas las armas alcanzó 2,62 mmol/lt. En el arma de la Espada se encuentra la muestra con el valor más bajo de producción de lactato, correspondiente a $1,89 \mathrm{mmol} / \mathrm{lt}$, mientras que el valor más alto obtenido de entre todas las muestras se encuentra en el arma de Florete, correspondiente a 3,46 mmol/lt. También se puede observar que el arma de la Espada posee el promedio más bajo de producción de lactato sanguíneo, con una media de 2,46 mmol/lt; seguido por el arma del Sable con una media de 2,67 mmol/lt; mientras que el arma del Florete posee el nivel más alto de producción de lactato sanguíneo, con un promedio de $2,73 \mathrm{mmol} / \mathrm{lt}$. Sin embargo, al comparar los valores de lactato sanguíneo entre las tres armas, se establece que no existen diferencias estadísticamente significativas entre ellas $(F(2,21)$ $=1,2937 ; p=0,295)$.

Tabla I: Resultados descriptivos de la concentración de lactato sanguíneo (mmol/lt) según arma.

\begin{tabular}{cccccc}
\hline Arma & N & $\begin{array}{c}\text { Media } \\
(\mathrm{mmol} / \mathrm{lt})\end{array}$ & DS & Mín. & Máx. \\
\hline Espada & 8 & 2,46 & 0,36 & 1,89 & 2,90 \\
Florete & 8 & 2,73 & 0,37 & 2,33 & 3,46 \\
Sable & 8 & 2,67 & 0,35 & 2,18 & 3,17 \\
Todas & $\mathbf{2 4}$ & $\mathbf{2 , 6 2}$ & $\mathbf{0 , 3 6}$ & $\mathbf{1 , 8 9}$ & $\mathbf{3 , 4 6}$ \\
\hline
\end{tabular}


BAHAMONDES-AVILA, C.; CÁRCAMO-OYARZÚN, J. \& HERNÁNDEZ-MOSQUEIRA, C. (2014). Concentraciones máximas de lactato sanguíneo producidos en combates de esgrima dependiendo del tipo de arma. Mot. Hum. 15(2): 116-123.

\section{DISCUSIÓN}

Los resultados de este estudio se encuentran dentro de los rangos obtenidos en otros trabajos relacionados a la producción de lactato en combates de esgrima. La media de las tres armas $(2,62$ $\mathrm{mmol} / \mathrm{lt})$, así como los valores mínimo $(1,89$ $\mathrm{mmol} / \mathrm{lt})$ y máximo $(3,46 \mathrm{mmol} / \mathrm{lt})$ se encuentran muy cercanos a los resultados obtenidos por (Cerizza \& Roi, 1994), quienes midieron las concentraciones de lactato en una competencia de nivel regional de florete masculino, obteniendo una media de 2,5 mmol/lt (1,4 mmol/lt mínimo y 3,9 mmol/lt como máximo); sin embargo es necesario considerar que las muestras fueron obtenidas después de cinco minutos de finalizado los combates durante la primera fase del campeonato, lo que podría significar que las concentraciones pudiesen haber sido un poco más altas si se hubiese realizado la medición un minuto después de finalizado los combates, como la metodología utilizada en el presente estudio.

Otra investigación que midió las concentraciones de lactato en competencias oficiales de esgrima es la de (Iglesias \& Rodríguez, 1995; Iglesias \& Rodríguez, 1999), quienes analizaron la lactacidemia en 66 asaltos de espada masculina y florete femenino, tomando las muestras al minuto uno y al minuto tres después de finalizado el asalto. Los resultados son relativamente más altos que los de los esgrimistas chilenos, con una media de $3,7 \mathrm{mmol} / \mathrm{lt}$ (3,2 $\mathrm{mmol} / \mathrm{lt}$ para espada masculina y 4,2 mmol/lt para florete femenino).

Valores de concentraciones de lactato obtenidos en condiciones de combates de entrenamiento también se acercan a los resultados del presente estudio; (Bottoms, 2011), analizaron las concentraciones de lactato del equipo nacional de espada femenino de Polonia al final de combates simulados de tres minutos, obteniendo una media de $2,8 \mathrm{mmol} / \mathrm{lt}$. (Weichenberger et al., 2012) midió las concentraciones de lactato en combates simulados en espada y florete, tanto de damas como varones, tomando las muestras una vez finalizado cada combate, obteniendo una media de $1,7 \mathrm{mmol} / \mathrm{lt}(0,9$ mmol/lt como minimo y $2,5 \mathrm{mmol} / \mathrm{lt}$ como valor máximo). A pesar de la posible similitud de los resultados, queda en evidencia que no existe una metodología uniforme para la valoración de las concentraciones de lactato, lo que hace difícil una comparación directa entre los estudios anteriormente expuestos, principalmente dado por la diferencia entre diversas variables relevantes como el nivel de rendimiento de los deportistas; la fase de la competencia; y el tiempo transcurrido entre el termino del combate y la toma de la muestra sanguínea. El nivel de experiencia de los esgrimistas influiría en el desempeño durante los combates, ya que los esgrimistas que participan en competencias internacionales, considerados de elite, tendrían un mejor rendimiento físico que los esgrimistas locales considerados como no de elite (Roi, Fiore, Bressan, Gatti \& Pittaluga, 2002; Weichenberger et al., 2012). Asimismo, cuando las capacidades técnico - táctico de un tirador son mejores que las del adversario, la participación del metabolismo es alto pero siempre submáximo (Roi et al., 2002). La fase de la competencia en que se realizan las mediciones también condicionaría las concentraciones de lactato, en donde a medida que avanza un torneo se produciría un aumento de la lactacidemia, debido a la acción del sistema nervioso simpático, existiendo una relación entre el flujo neto de lactato muscular y la concentración de adrenalina arterial, independiente del oxígeno inspirado (Hoch, Werle, \& Weicker, 1988; Richardson, Noyszewski, Leigh, \& Wagner, 1998). A medida que un tirador va avanzando en un torneo, los combates tendrán una mayor exigencia técnico táctica, lo que aumentaría la participación metabólica y muscular, y el rol del metabolismo láctico se volvería más importante (Roi et al., 2002). También influirían en las concentraciones de lactato el momento en el que se toma la muestra después del combate, ya que la tasa de aclaramiento va a diferir si la muestra sanguínea se recolecta inmediatamente terminado el combate o si se dejan transcurrir varios minutos; causas posibles que podrían ejercer su influencia en este proceso son el tipo de fibra muscular, la actividad enzimática de la vía glicolítica o lipolítica, la capilarización del músculo, la densidad mitocondrial, el trasportador de lactato (MCT) o las adaptaciones al entrenamiento, las cuales deben ser tomadas en cuenta al momento de interpretar los resultados (Faude et al., 2009; Menzies et al., 2010), dado que aún la comprensión de la remoción del lactato después de un ejercicio de alta intensidad a del cuerpo entero requiere un análisis más detenido (Moxnes \& Sandbakk, 2012). 
BAHAMONDES-AVILA, C.; CÁRCAMO-OYARZÚN, J. \& HERNÁNDEZ-MOSQUEIRA, C. (2014). Concentraciones máximas de lactato sanguíneo producidos en combates de esgrima dependiendo del tipo de arma. Mot. Hum. 15(2): 116-123.

Otro factor al que hay que prestar atención y que podría mal interpretar la información sobre el comportamiento del lactato en ejercicio es el agotamiento de las reservas de glucógeno, ya sea por una baja ingesta previa de hidratos de carbono o por la realización de un ejercicio extenuante (Shiose, Tobina, Higaki, Kiyonaga, \& Tanaka, 2012); donde las concentraciones de lactato sanguíneo son inferiores a las mismas tasas de trabajo al comparar sujetos con el glucógeno depletado en relación a sujetos en condiciones normales (Langfort, Czarnowski, EndzianPiotrowska, Zarzecny, \& Górski, 2004; Maassen \& Busse, 1989).

Sin embargo, independiente de la metodología utilizada para estimar las concentraciones máximas de lactato en combates de esgrima, tanto el presente trabajo como la mayoría de las investigaciones referente a este tema reportan que no se produce una alta concentración de lactato después de combates de esgrima. Si se comparan estos resultados con los valores de referencia de los diversos conceptos relacionados con el inicio de la producción de lactato sanguíneo en ejercicio, así como de la cinética del lactato en ejercicios incrementales, estos valores son considerados como bajos y previos al aumento exponencial del lactato (Calderón Montero, Benito Peinado, Peinado Lozano, \& Díaz Molina, 2008; Faude et al., 2009; Péronnet, 2010), por lo que los resultados de este estudio reflejan que, independiente del tipo de arma, existe una escasa participación del sistema anaeróbico láctico durante los combates de esgrima. Esto se podría explicar debido a la intermitencia de las acciones en el combate, pues durante la competencia se producen múltiples pausas, debido a las puestas en guardia cada vez que se termina una acción de ataque y por los tiempos reglamentarios de descanso (uno de descanso por tres de combate); lo que permitiría un periodo de recuperación, permitiendo una resíntesis rápida de ATP a expensas de la fosfocreatina entre picos de activación intensa del metabolismo anaeróbico y de resíntesis más lenta a cargo de la fosforilación oxidativa (Iglesias \& Rodríguez, 1995; Péronnet, 2010). A ello se debe sumar un aumento en la tasa de aclaramiento del ácido láctico producido post competencia, favorecido por un estado de metabolismo aeróbico, permitiendo la utilización del ácido láctico como intermediario metabólico y sustrato energético, ligados a la gluconeogénesis (Emhoff, 2013; Facey, Irving, \& Dilworth, 2013).

Finalmente este estudio determinó las concentraciones máximas de lactato en una fase específica de una competencia oficial de carácter Nacional, entregando valores que indican una escasa participación del sistema anaeróbico láctico. Asimismo se determinó que, a pesar de las diferencias técnicas y reglamentarias que existen, el tipo de arma no influiría en las concentraciones de ácido láctico producidas en combate, estableciendo que tanto la espada como el sable y el florete requieren un alto nivel de acondicionamiento aeróbico y anaeróbico (Ghloum \& Hajji, 2011; Iglesias \& Rodríguez, 1995; Weichenberger et al., 2012). Este sería el primer reporte en una competencia oficial de esgrima en Chile y por lo anteriormente expuesto, los resultados de este estudio toman relevancia y servirían como herramienta para mejorar la aplicación de las cargas físicas y selección de los tipos o métodos de entrenamiento, los que deben considerar la predominancia de los sistemas energéticos anaeróbico aláctico o de ATP - PC y aeróbico u oxidativo (Weichenberger et al., 2012; Wylde et al., 2013).

\section{CONCLUSIONES}

Este estudio determinó las concentraciones máximas de lactato en una fase específica de una competencia oficial, entregando valores que indican una escasa participación del sistema anaeróbico láctico. Asimismo se determinó que, a pesar de las diferencias técnicas y reglamentarias que existen, el tipo de arma no influiría en las concentraciones de ácido láctico producidas en combate, estableciendo que tanto la espada como el sable y el florete requieren un alto nivel de acondicionamiento aeróbico y anaeróbico. 
BAHAMONDES-AVILA, C.; CÁRCAMO-OYARZÚN, J. \& HERNÁNDEZ-MOSQUEIRA, C. (2014). Concentraciones máximas de lactato sanguíneo producidos en combates de esgrima dependiendo del tipo de arma. Mot. Hum. 15(2): 116-123.

\section{REFERENCIAS BIBLIOGRAFICAS}

Bottoms, L. (2011). Physiological responses and energy expenditure to simulated epee fencing in elite female fencers. Serbian journal of sports sciences, 5(1), 17-20.

Calderón Montero, F. J., Benito Peinado, P. J., Peinado Lozano, A. B., \& Díaz Molina, V. (2008). Significado fisiológico de la transición aeróbica-anaeróbica. Revista Internacional de Medicina y Ciencias de la Actividad Física y el Deporte, 8(32), 321337.

Cerizza, C., \& Roi, G. (1994). Aspetti fisiologici dell'attività sportiva di base, le caratteristiche fondamentali del giovane schermidore. Sport \& educazione giovanile. Milan: Ghedini Editore, 89-96.

Dassonville, J., Beillot, J., Lessard, Y., Jan, J., Andre, A., Le Pourcelet, C., . . . Carre, F. (1998). Blood lactate concentrations during exercise: effect of sampling site and exercise mode. J Sports Med Phys Fitness, 38(1), 3946.

Emhoff, C.-A. W. (2013). Regulation of Glycemia and Energy Substrate Partitioning in Exercising Humans. Tesis Doctoral. Berkeley, University of California.

Facey, A., Irving, R., \& Dilworth, L. (2013). Overview of Lactate Metabolism and the Implications for Athletes. Nature, 1(3), 4246.

Faude, O., Kindermann, W., \& Meyer, T. (2009). Lactate threshold concepts: how valid are they? Sports Medicine (Auckland, N.Z.), 39(6), 469-490. doi: 10.2165/00007256200939060-00003

Gharbi, A., Chamari, K., Kallel, A., Ahmaidi, S., Tabka, Z., \& Abdelkarim, Z. (2008). Lactate kinetics after intermittent and continuous exercise training. Journal of sports science \& medicine, 7(2), 279.

Ghloum, K., \& Hajji, S. (2011). Comparison of diet consumption, body composition and lipoprotein lipid values of Kuwaiti fencing players with international norms. J Int Soc Sports Nutr, 8(1), 1-9.

Hoch, F., Werle, E., \& Weicker, H. (1988). Sympathoadrenergic regulation in elite fencers in training and competition. Int $J$ Sports Med, 9(S 2), 141-145.

Iglesias, X., Irurtia, A., Marina, M., \& Carrasco, M. Dèficits bilaterals i asimetries morfofuncionals dels esgrimidors joves Déficits bilaterales y asimetrías morfofuncionales en jóvenes esgrimistas Bilateral deficit and morphofunctional asymmetries in young fencers.

Iglesias, X., \& Rodríguez, F. (1995). Caracterización de la frecuencia cardiaca y la Iactatemia en esgrimistas durante la competición. Apunts. Medicina de l'Esport, 32(123), 21-32.

Iglesias, X., \& Rodríguez, F. (2000). Consumo de Oxígeno en Asaltos de Esgrima Valoracación Directa y Validación de un Método de Estimación. Apunts. Medicina de l'Esport, 35(133), 29-36.

Iglesias, X., \& Rodríguez, F. A. (1999). Consumo de oxígeno estimado y gasto energético en competiciones de esgrima.

Langfort, J., Czarnowski, D., Endzian-Piotrowska, M., Zarzecny, R., \& Górski, J. (2004). Short-term low-carbohydrate diet dissociates lactate and ammonia thresholds in men. The Journal of Strength \& Conditioning Research, 18(2), 260-265.

Maassen, N., \& Busse, M. (1989). The relationship between lactic acid and work load: a measure for endurance capacity or an indicator of carbohydrate deficiency? European journal of applied physiology and occupational physiology, 58(7), 728-737.

Marginet, M. C., Amigó, A. I., Sala, V. P., Reig, X. I., García, E. V., \& Brotons, D. (2008). Valoración nutricional de los hábitos alimentarios en jóvenes esgrimistas de competición. Apunts. Medicina de l'Esport, 43(159), 118-126.

Menzies, P., Menzies, C., McIntyre, L., Paterson, P., Wilson, J., \& Kemi, O. J. (2010). Blood lactate clearance during active recovery after an intense running bout depends on the intensity of the active recovery. $J$ Sports $S c i$, 28(9), 975-982.

Moxnes, J. F., \& Sandbakk, K. (2012). The kinetics of lactate production and removal during whole-body exercise. Theor Biol Med Model, 9(7). 
Péronnet, F. (2010). Lactate as an end-product and fuel. Deutsche Zeitschrift fur Sportmedizin, 61(5), 112.

Richardson, R. S., Noyszewski, E. A., Leigh, J. S., \& Wagner, P. D. (1998). Lactate efflux from exercising human skeletal muscle: role of intracellular. Journal of Applied Physiology, 85(2), 627-634.

Roi, G. S., \& Bianchedi, D. (2008). The science of fencing: implications for performance and injury prevention. Sports Medicine, 38(6), 465-481.

Roi G.S., T. G., Fiore A, Bressan A, Gatti M, Pittaluga. (2002). Zur Entwicklung eines Leistungsmodells im Spitzensport am Beispiel Fechten. Leistungssport, 32, 57-62.

Ryan, T. E., Southern, W. M., Reynolds, M. A., \& McCully, K. K. (2013). A cross-validation of near-infrared spectroscopy measurements of skeletal muscle oxidative capacity with phosphorus magnetic resonance spectroscopy. Journal of Applied Physiology, 115(12), 1757-1766.

Santhiago, V., Silva, A. S. R. d., Guglielmo, L. G. A., \& Higino, W. P. (2008). Influência da forma de indução à acidose na determinação da intensidade de lactato mínimo em corredores de longa distância. Rev. bras. med. esporte, 14(4), 393-398.
Shiose, K., Tobina, T., Higaki, Y., Kiyonaga, A., \& Tanaka, H. (2012). Effectiveness of submaximal intermittent exercise on muscle glycogen depletion, PGC-1 $\alpha$ and PDK-4 gene expression. Open Journal of Molecular and Integrative Physiology, 2, 119.

Souza, K. M. d., Grossl, T., Babel Junior, R. J., Lucas, R. D. d., Costa, V. P., \& Guglielmo, L. G. A. (2012). Máximo estado estável de lactato estimado por diferentes métodos de determinação do limiar anaeróbio; Maximal lactate steady state estimated by different methods of anaerobic threshold. Rev. Bras. Cineantropom. Desempenho Hum, 14(3), 264-275.

Tomlin, D. L., \& Wenger, H. A. (2001). The relationship between aerobic fitness and recovery from high intensity intermittent exercise. Sports Medicine, 31(1), 1-11.

Weichenberger, M., Liu, Y., \& Steinacker, J. (2012). A test for determining endurance capacity in fencers. Int $J$ Sports Med, 33(01), 48-52.

Wylde, M. J., Tan, F. H., \& O'Donoghue, P. G. (2013). A time-motion analysis of elite women's foil fencing. International Journal of Performance Analysis in Sport, 13(2), 365-376.

\section{Enviar Correspondencia a:}

Carlos Bahamondes-Avila

Universidad Mayor, Sede Temuco

Avenida Alemania 0281, Temuco, Chile

Email - carlos.bahamondesa@mayor.cl 